\title{
Sharps Bounds for Power Mean in Terms of Contraharmonic Mean
}

\section{Zeid I. Almuhiameed}

Department of Mathematics, College of Sciences, Qassim University, Al-Mulida, Qassim, KSA

Email: ksapr006@yahoo.com

How to cite this paper: Almuhiameed, Z.I. (2020) Sharps Bounds for Power Mean in Terms of Contraharmonic Mean. Journal of Applied Mathematics and Physics, 8, 1229-1235.

https://doi.org/10.4236/jamp.2020.87093

Received: June 3, 2020

Accepted: July 5, 2020

Published: July 8, 2020

Copyright $\odot 2020$ by author(s) and Scientific Research Publishing Inc. This work is licensed under the Creative Commons Attribution International License (CC BY 4.0).

http://creativecommons.org/licenses/by/4.0/

\section{Abstract}

In this research work, we consider the below inequalities: (1.1). The researchers attempt to find an answer as to what are the best possible parameters $\alpha, \beta$ that (1.1) can be held? The main tool is the optimization of some suitable functions that we seek to find out. Without loss of generality, we have assumed that $a>b$ and let $t=\frac{a}{b}>1$ for 1$)$ and $a<b, t=\frac{a}{b} \ll 1 \quad(t$ small) for 2) to determine the condition for $\alpha$ and $\beta$ to become $f(t) \leq 0$ and $g(t) \geq 0$.

\section{Keywords}

Sharps Bounds, Power Mean, Contraharmonic Mean

\section{Introduction}

The main objective of this research work is to present optimization of inequality in the one-parameter, arithmetic and harmonic means as follows:

$$
C[\alpha a+(1-\alpha) b, \alpha b+(1-\alpha) a] \leq M_{p}(a, b) \leq C[\beta a+(1-\beta) b, \beta b+(1-\beta) a]
$$

with $C(a, b)=\frac{a^{2}+b^{2}}{a+b}$ and

$$
M_{p}(a, b)= \begin{cases}\left(\frac{a^{P}+b^{P}}{2}\right)^{\frac{1}{p}} ; & p \neq 0 \\ \sqrt{a b}, & p=0\end{cases}
$$

Our motivation for this study is to find out such inequality that arises in the search for determination of a point of reference about which some function of 
variants would be minimum or maximum. Since very early times, people have been interested in the problem of choosing the best single quantity, which could summarize the whole information contained in a number of observations (measurements). Moreover, the theory of means has its roots in the work of the Pythagorean who introduced the harmonic, geometric, and arithmetic means. The strong relations and introduction of the theory of means with the theories of inequalities, function equations, probability and statistics add greatly to its importance. This single element is usually called a means or averages. The term "means" or "average" (middle value) has for a long time been used in all branches of human activity.

The basic function of mean value is to represent a given set of many values by some single value. In [1], the authors for the first time introduced power means defining the meaning of the term "representation" as determination of appointing of reference about which some function of variants would be minimum. More recently the means were the subject of research, study, and essential areas in several applications such as physics, economics, electrostatics, heat conduction, medicine, and even in meteorology. It can be observed the power mean $M_{p}(a, b)$ (see as [2]).

If we denote by

$$
A(a, b)=\frac{1}{2}(a+b), G(a, b)=\sqrt{a b} \text { and } H(a, b)=\frac{2 a b}{a+b},
$$

the arithmetic means, geometric means and harmonic means of two positive numbers $a$ and $b$, respectively. In addition, the logarithmic and identric means of two positive real numbers $a$ and $b$ were defined by [3]

$$
\begin{aligned}
& L(a, b)= \begin{cases}\frac{b-a}{\log b-\log a} & a \neq b \\
a & a=b\end{cases} \\
& I(a, b)= \begin{cases}\frac{1}{e}\left(\frac{b^{b}}{a^{a}}\right)^{1 l(b-a)} & a \neq b \\
a & a=b\end{cases}
\end{aligned}
$$

Several authors investigated and developed relationship of optimal inequalities between the various means.

The well-known inequality that:

$$
\begin{aligned}
\min \{a, b\} & \leq H(a, b)=M_{-1}(a, b) \\
& \leq G(a, b)=M_{0}(a, b) \\
& \leq L(a, b) \leq I(a, b) \leq A(a, b)=M_{1}(a, b) \leq \max \{a, b\}
\end{aligned}
$$

and all inequalities are strict for $a \neq b$.

In [3], researchers studied what are the best possible parameters $\alpha_{1}, \alpha_{2}, \beta_{1}$ and $\beta_{2}$ by two theorems:

Theorem (1) the double inequality: 


$$
\alpha_{1} A(a, b)+\left(1-\alpha_{1}\right) H(a, b) \leq L(a, b) \leq \beta_{1} A(a, b)+\left(1-\beta_{1}\right) H(a, b)
$$

holds for all $a, b>0$ if and only if $\alpha_{1} \leq 0$ and $\beta_{1} \geq \frac{2}{3}$ when proved that the parameters $\alpha_{1} \leq 0$ and $\beta_{1} \geq \frac{2}{3}$ cannot be improved.

Theorem (2) the double inequality:

$$
\alpha_{2} A(a, b)+\left(1-\alpha_{2}\right) H(a, b) \leq L(a, b) \leq \beta_{2} A(a, b)+\left(1-\beta_{2}\right) H(a, b)
$$

holds for all $a, b>0$ if and only if $\alpha_{2} \leq \frac{2}{e}$ and $\beta_{2} \geq \frac{5}{6}$ when proved that the parameters $\alpha_{2} \leq \frac{2}{e}$ and $\beta_{2} \geq \frac{5}{6}$ cannot be improved; holds for all $a, b>0$ with $a \neq b$, and they found $L_{2}(a, b)$ the optimal lower generalized logarithmic means bound for the identric means $I(a, b)$ for inequalities $L_{2}(a, b)<I(a, b)$; holds for all $a, b$ are positive numbers with $a \neq b$. Pursuing another line of investigation, in [4] the authors showed the sharp upper and lower bounds for the Neuman-Sandor $N S(a, b)$ [5] in terms of the linear convex combination of the logarithmic means $L(a, b)$, and second Seiffert means $T(a, b)$ [6] of two positive numbers $a$ and $b$, respectively for the double inequalities

$$
\alpha L(a, b)+(1-\alpha) T(a, b) \leq N S(a, b) \leq \beta L(a, b)+(1-\beta) T(a, b)
$$

holds for all $a, b>0$ with $a \neq b$ is true if and only if $\alpha \geq \frac{1}{4}$ and $\beta \leq 1-\pi l[4 \log (1+\sqrt{2})]$.

In [7], $\mathrm{HZ} \mathrm{Xu}$ et al. have improvements and refinements, for they found several sharp upper and lower bounds for the Sandor-Yang means $R_{Q A}(a, b)$ and $R_{A Q}(a, b)$. In terms of combinations of the arithmetic means $A(a, b)$, there is [8]; and in terms of the contraharmonic mean $C(a, b)$, there is [9].

\section{Main Results}

Our main results are set in the following theorem:

Theorem 1. Assume $a>0, b>0$ then, there exist $\Lambda_{1}, \Lambda_{2}, \Lambda_{3}, \Lambda_{4}$ reals such that

1) If $p \in(-\infty,-3), \alpha \in\left(\Lambda_{1}, \Lambda_{2}\right)$ and $\beta \in\left(-\infty, \Lambda_{1}\right) \cup\left(\Lambda_{2},+\infty\right)$ with $\frac{a}{b}>1$ then, the double inequality (1.1) holds.

2) If $p=0, \quad \alpha \in(-\infty, 0) \cup(1,+\infty)$ and $\beta \in\left(\Lambda_{3}, \Lambda_{4}\right)$ with $\frac{a}{b} \ll 1 \quad\left(\frac{a}{b}\right.$ small) then the double inequality (1.1) holds.

Proof. 1) Assuming $a>0, b>0$ with $\frac{a}{b}>1$

We have

$$
\begin{aligned}
& \frac{[\alpha a+(1-\alpha) b]^{2}+[\alpha b+(1-\alpha) a]^{2}}{[\alpha a+(1-\alpha) b]+[\alpha b+(1-\alpha) a]} \\
& \leq\left(\frac{a^{P}+b^{P}}{2}\right)^{\frac{1}{p}} \leq \frac{[\beta a+(1-\beta) b]^{2}+[\beta b+(1-\beta) a]^{2}}{[\beta a+(1-\beta) b]+[\beta b+(1-\beta) a]}, p \neq 0 ; a>b .
\end{aligned}
$$

Set $t=\frac{a}{b}>1$. Then, we obtain 


$$
\frac{b 2^{\frac{1}{p}}\left[\left(2 \alpha^{2}-2 \alpha+1\right) t^{2}+\left(4 \alpha-4 \alpha^{2}\right) t+\left(2 \alpha^{2}-2 \alpha+1\right)\right]-b(t+1)\left(t^{p}+1\right)^{\frac{1}{p}}}{2^{\frac{1}{p}}(t+1)} \leq 0
$$

and

$$
\frac{b 2^{\frac{1}{p}}\left[\left(2 \beta^{2}-2 \beta+1\right) t^{2}+\left(4 \beta-4 \beta^{2}\right) t+\left(2 \beta^{2}-2 \beta+1\right)\right]-b(t+1)\left(t^{p}+1\right)^{\frac{1}{p}}}{2^{\frac{1}{p}}(t+1)} \geq 0
$$

Because $b>0$ and $2^{\frac{1}{p}}(t+1)>0$ therefore the study amounts to proving that

$$
2^{\frac{1}{p}}\left[\left(2 \alpha^{2}-2 \alpha+1\right) t^{2}+\left(4 \alpha-4 \alpha^{2}\right) t+\left(2 \alpha^{2}-2 \alpha+1\right)\right]-(t+1)\left(t^{p}+1\right)^{\frac{1}{p}} \leq 0 .
$$

Let

$$
f(t)=2^{\frac{1}{p}}\left[\left(2 \alpha^{2}-2 \alpha+1\right) t^{2}+\left(4 \alpha-4 \alpha^{2}\right) t+\left(2 \alpha^{2}-2 \alpha+1\right)\right]-(t+1)\left(t^{p}+1\right)^{\frac{1}{p}}
$$

We have to prove that the function $f$ is negative under certain conditions on the parameters $\alpha, \beta$ and $p$, a.e: $f(t) \leq 0$. So

$$
\begin{aligned}
f(t)= & 2^{\frac{1}{p}}\left[\left(2 \alpha^{2}-2 \alpha+1\right) t^{2}+\left(4 \alpha-4 \alpha^{2}\right) t+\left(2 \alpha^{2}-2 \alpha+1\right)\right] \\
& -(t+1)\left(t^{p}+1\right)^{\frac{1}{p}} \leq 0 .
\end{aligned}
$$

Because $f(1)=0$, it will suffice to show that $f$ is decreasing for all $t>1$, which amounts to studying the sign of the derivative $f^{\prime}$ of $f$. We have:

$$
\begin{aligned}
f^{\prime}(t)= & 2^{\frac{1}{p}}\left(4 \alpha^{2}-4 \alpha+2\right) t+2^{\frac{1}{p}}\left(4 \alpha-4 \alpha^{2}\right) \\
& -\left[\left(t^{p}+1\right)^{\frac{1}{p}}+\left(t^{p}+t^{p-1}\right)\left(t^{p}+1\right)^{\frac{1}{p}-1}\right]
\end{aligned}
$$

Because $f^{\prime}(1)=0$, it will suffice to show that $f^{\prime}$ is decreasing for all $t>1$, which amounts to studying the sign of the derivative $f^{\prime \prime}$ of $f^{\prime}$. We have:

$$
\begin{aligned}
f^{\prime \prime}(t)= & 2^{\frac{1}{p}}\left(4 \alpha^{2}-4 \alpha+2\right)-\left[t^{p-1}\left(t^{p}+1\right)^{\frac{1}{p}-1}\left(p t^{p-1}+(p-1) t^{p-2}\right)\left(t^{p}+1\right)^{\frac{1}{p}-1}\right] \\
& -\left[(1-p)\left(t^{2 p-1}+t^{2 p-2}\right)\left(t^{p}+1\right)^{\frac{1}{p}-2}\right] \\
& +[\alpha b p(p+1)(p-1)] t^{p-2}+[-2 \alpha b(p+1)]
\end{aligned}
$$

There exixt

$$
\Lambda_{1}=\frac{2-\sqrt{2(-3-p)}}{4} \text { and } \Lambda_{2}=\frac{2+\sqrt{2(-3-p)}}{4}
$$

with $p<-3$ such that $f^{\prime \prime}(1)<0$ for all $\alpha \in\left(\Lambda_{1}, \Lambda_{2}\right)$ so it will suffice to show that $f^{\prime \prime}$ is decreasing for all $t>1$, which amounts to studying the sign of the derivative $f^{\prime \prime \prime}$ of $f^{\prime \prime}$. We have: 


$$
\begin{aligned}
f^{\prime \prime \prime}(t)= & -t^{p-3}\left(t^{p}+1\right)^{\frac{1}{p}-3}\left[\left(3 p^{2}-3 p\right) t^{2 p+1}+\left(3 p^{2}-6 p+3\right) t^{2 p}\right. \\
& +(1-p) t^{p+2}+\left(-p^{2}+4 p-3\right) t^{p+1}+\left(1-p^{2}\right) t^{p} \\
& \left.+(1-p) t^{2}+\left(p^{2}-1\right) t+(p-1)(p-2)\right]
\end{aligned}
$$

and we get

$$
f^{\prime \prime \prime}(1)<0
$$

and since $p<-3$ we obtain that

$$
\left(3 p^{2}-3 p\right)>-\left(-p^{2}+4 p-3\right) \text { and }\left(3 p^{2}-6 p+3\right)>\left(p^{2}-1\right)
$$

so, we will have

$$
f^{\prime \prime \prime}(t)<0 \text { for all } t \geq 1
$$

By the same process we find that $f^{\prime \prime}(t)<0$ then that $f^{\prime}(t)<0$ and $f(t)<0$.

Finally in this part there exixt

$$
\Lambda_{1}=\frac{2-\sqrt{2(-3-p)}}{4} \text { and } \Lambda_{2}=\frac{2+\sqrt{2(-3-p)}}{4}
$$

with $p<-3$ such that for all $\alpha \in\left(\Lambda_{1}, \Lambda_{2}\right)$ we have:

$$
\frac{b 2^{\frac{1}{p}}\left[\left(2 \alpha^{2}-2 \alpha+1\right) t^{2}+\left(4 \alpha-4 \alpha^{2}\right) t+\left(2 \alpha^{2}-2 \alpha+1\right)\right]}{2^{\frac{1}{p}}(t+1)}-\frac{b(t+1)\left(t^{p}+1\right)^{\frac{1}{p}}}{2^{\frac{1}{p}}(t+1)} \leq 0,
$$

i.e.:

$$
C[\alpha a+(1-\alpha) b, \alpha b+(1-\alpha) a] \leq M_{p}(a, b)
$$

To show the second inequality in this first case, we proceed by similar calculations. This is done by considering the function $g$ defined by

$$
g(t)=2^{\frac{1}{p}}\left[\left(2 \beta^{2}-2 \beta+1\right) t^{2}+\left(4 \beta-4 \beta^{2}\right) t+\left(2 \beta^{2}-2 \beta+1\right)\right]-(t+1)\left(t^{p}+1\right)^{\frac{1}{p}}
$$

So, after all the calculations, we get that for $p<-3$ and $\beta \in\left(-\infty, \Lambda_{1}\right) \cup\left(\Lambda_{2},+\infty\right): g(t) \geq 0$. a.e:

$$
M_{p}(a, b) \leq C[\beta a+(1-\beta) b, \beta b+(1-\beta) a]
$$

2) With similar calculations and by the same idea we obtain that if $p=0$, $\alpha \in(-\infty, 0) \cup(1,+\infty)$ and $\beta \in\left(\Lambda_{3}, \Lambda_{4}\right)$ with

$$
\Lambda_{3}=\frac{1+\sqrt{3}}{2}, \Lambda_{4}=\frac{1-\sqrt{3}}{2}
$$

and $\frac{a}{b} \ll 1\left(\frac{a}{b}\right.$ small $)$ then,

$$
C[\alpha a+(1-\alpha) b, \alpha b+(1-\alpha) a] \leq M_{p}(a, b) \leq C[\beta a+(1-\beta) b, \beta b+(1-\beta) a] .
$$

Conclusion 1. In our work, we studied the following double inequality 


$$
C[\alpha a+(1-\alpha) b, \alpha b+(1-\alpha) a] \leq M_{p}(a, b) \leq C[\beta a+(1-\beta) b, \beta b+(1-\beta) a] .
$$

by searching the best possible parameters such that (1.1) can be held.

Firstly, we have inserted

$$
f(t)=C[\alpha a+(1-\alpha) b, \alpha b+(1-\alpha) a]-M_{p}(a, b)
$$

Without loss of generality, we have assumed that $a>b$ and let $t=\frac{a}{b}>1$ for 1 ) and $a<b, t=\frac{a}{b} \ll 1$ ( $t$ small) for 2) to determine the condition for $\alpha$ and $\beta$ to become $f(t) \leq 0$.

\section{Secondly, have inserted}

$$
g(t)=C[\beta a+(1-\beta) b, \beta b+(1-\beta) a]-M_{p}(a, b)
$$

Without loss of generality, we assume that $a>b$ and let $t=\frac{a}{b}>1$ for 1 ) and $a<b, t=\frac{a}{b} \ll 1$ ( $t$ small) for 2 ) to determine the condition for $\alpha$ and $\beta$ to become $g(t) \geq 0$.

And finally, we got that:

1) if $p \in(-\infty,-3), \alpha \in\left(\Lambda_{1}, \Lambda_{2}\right)$ and $\beta \in\left(-\infty, \Lambda_{1}\right) \cup\left(\Lambda_{2},+\infty\right)$ with $\frac{a}{b}>1$ then, the double inequality (1.1) holds,

2) if $p=0, \quad \alpha \in(-\infty, 0) \cup(1,+\infty)$ and $\beta \in\left(\Lambda_{3}, \Lambda_{4}\right)$ with $\frac{a}{b} \ll 1 \quad\left(\frac{a}{b}\right.$ small) then the double inequality (1.1) holds.

\section{Conflicts of Interest}

The author declares no conflicts of interest regarding the publication of this paper.

\section{References}

[1] Ostasiewicz, S. and Ostasiewicz, W. (2000) Means and Their Applications. Annals of Operations Research, 97, 337-355. https://doi.org/10.1023/a:1018932425645

[2] Long, B.Y., Li, Y.M. and Chu, Y.M. (2012) Optimal Inequalities between Generalized Logarithmic, Identric and Power Means. International Journal of Pure and Applied Mathematics, 80, 41-51.

[3] Xia, W.-F. and Chu, Y.-M. (2010) Optimal Inequalities Related to the Logarithmic, Identric, Arithmetic and Harmonic Means. Revue d Analyse Numerique et de Theorie de 1 Approximation, 39, 176-183.

[4] Chen, J.-J., Lei, J.-J. and Long, B.-Y. (2017) Optimal Bounds for Neuman-Sandor Means in Term of the Convex Combination of the Logarithmic and the Second Seiffert Means. Journal of Inequalities and Applications, No. 1, 251. https://doi.org/10.1186/s13660-017-1516-7

[5] Newman, E. and Sandor, J. (2003) On the Schwab-Borcharrdt Means. Mathematica Pannonica, 14, 253-266.

[6] Seiffert, S., Kaselowesky, J., Jungk, A. and Claassen, N. (1995) Observed and Calculated Potassium Uptake by Maize as Affected by Soil Water Content and Bulk Density. Agronomy Journal, 87, 1070-1077. https://doi.org/10.2134/agronj1995.00021962008700060007x 
[7] Xu, H.-Z., Chu, Y.-M. and Qian, W.-M. (2018) Sharp Bounds for the Sandor-Yang Means in Terms of Arithmetic and Contra-Harmonic Means. Journal of Inequalities and Applications, No. 1, 127. https://doi.org/10.1186/s13660-018-1719-6

[8] Yang, Y.Y. and Qian, W.M. (2016) Two Optimal Inequalities Related to the Sandor-Yang Mean and One-Parameter Mean. Communications in Mathematical Research, 32, 352-358.

[9] Jiang, W.-D. and Qi, F. (2015) Sharp Bounds for the Neuman-Sandor Mean in Term of the Power and Contraharmonic Means. Cogent Mathematics, 2, 995951. https://doi.org/10.1080/23311835.2014.995951 